\title{
Integral Stability in Terms of Two Measures for Nonlinear Differential Systems with "Maxima"
}

\author{
Peiguang Wang, ${ }^{1}$ Qing Xu, ${ }^{2}$ and Xiaojing Liu ${ }^{2}$ \\ ${ }^{1}$ College of Electronic and Information Engineering, Hebei University, Baoding 071002, China \\ ${ }^{2}$ College of Mathematics and Computer Science, Hebei University, Baoding 071002, China \\ Correspondence should be addressed to Peiguang Wang; pgwang@hbu.edu.cn
}

Received 28 March 2014; Accepted 25 July 2014; Published 13 August 2014

Academic Editor: Tongxing Li

Copyright (c) 2014 Peiguang Wang et al. This is an open access article distributed under the Creative Commons Attribution License, which permits unrestricted use, distribution, and reproduction in any medium, provided the original work is properly cited.

\begin{abstract}
This paper investigates relatively integral stability in terms of two measures for two differential systems with "maxima" by employing
\end{abstract} Lyapunov functions, Razumikhin method, and comparison principle. An example is given to illustrate our result.

\section{Introduction}

Recently, the interest in differential equations with "maxima" has increased exponentially. Such equations adequately model real world problems whose present state depends significantly on its maximum value on a past time interval. For example, many problems in the control theory correspond to the maximal deviation of the regulated quantity. Some qualitative properties of the solutions of ordinary differential equations with "maxima" can be found in [1-4] and references therein.

Integral stability for ordinary differential equations was introduced by Vrkoc [5]. The concept of integral stability occurs in connection with the stability under persistent perturbations when the perturbations are small enough everywhere except on a small interval. Recent developments in this field have been focused on various types of differential equations. In $[6,7]$, the integral stability and integral $\phi_{0^{-}}$ stability properties of ordinary differential equations were discussed, respectively. Later, Hristova [8] discussed the integral stability in terms of two measures for impulsive differential equations with "supremum." Moreover the same author in [9] discussed the integral stability in terms of two measures for impulsive functional differential equations. However, the integral stability in terms of two measures for two differential systems has not been obtained until now.

In this paper, we discuss the relatively integral stability in terms of two measures for two differential systems with “maxima." Using Lyapunov functions, Razumikhin method, and comparison principle, sufficient conditions for uniformrelatively integral stability in terms of two measures are obtained.

\section{Preliminaries}

Firstly, we give the following sets for convenience:

$$
\begin{aligned}
& \mathscr{K}=\left\{a(t) \in C\left[R_{+}, R_{+}\right] \mid a(t)\right. \text { is strictly increasing, } \\
&a(0)=0\} ; \\
& \mathscr{C} \mathscr{K}=\left\{a(t) \in C\left[R_{+}^{2}, R_{+}\right] \mid \forall t \in R_{+}, a(t, u) \in \mathscr{K}\right\} ; \\
& \Gamma=\left\{h \in C\left[R_{+} \times R^{n}, R_{+}\right] \mid(t, x) \in R_{+} \times R^{n},\right. \\
& \\
&\quad \inf h(t, x)=0\} .
\end{aligned}
$$

Let $\rho$, t, and $r>0$ be constants, $h_{0} \in \Gamma$. Define the following sets:

$$
\begin{gathered}
S(h, \rho)=\left\{(t, x, y) \in R_{+} \times R^{n} \times R^{n} \mid h(t, x-y)<\rho\right\} ; \\
S^{c}(h, \rho)=\left\{(t, x, y) \in R_{+} \times R^{n} \times R^{n} \mid h(t, x-y) \geq \rho\right\} ; \\
\Omega(t, h, \rho)=\left\{(x, y) \in R^{n} \times R^{n} \mid h(t, x) \leq \rho,\right. \\
h(s, y) \leq \rho, s \in[t-r, t]\} .
\end{gathered}
$$


We consider the following two differential systems with "maxima":

$$
\begin{gathered}
x^{\prime}=F_{1}\left(t, x(t), \max _{s \in[t-r, t]} x(s)\right), \quad t \geq t_{0}, \\
x\left(t+t_{0}\right)=\phi_{1}(t), \quad t \in[-r, 0], \\
y^{\prime}=F_{2}\left(t, y(t), \max _{s \in[t-r, t]} y(s)\right), \quad t \geq t_{0}, \\
y\left(t+t_{0}\right)=\phi_{2}(t), \quad t \in[-r, 0],
\end{gathered}
$$

and the perturbed systems

$$
\begin{aligned}
x^{\prime}= & F_{1}\left(t, x(t), \max _{s \in[t-r, t]} x(s)\right) \\
& +G_{1}\left(t, x(t), \max _{s \in[t-r, t]} x(s)\right), \quad t \geq t_{0}, \\
& x\left(t+t_{0}\right)=\phi_{1}(t), \quad t \in[-r, 0], \\
y^{\prime}= & F_{2}\left(t, y(t), \max _{s \in[t-r, t]} y(s)\right) \\
& +G_{2}\left(t, y(t), \max _{s \in[t-r, t]} y(s)\right), \quad t \geq t_{0}, \\
& y\left(t+t_{0}\right)=\phi_{2}(t), \quad t \in[-r, 0],
\end{aligned}
$$

where $x, y \in R^{n}, F_{1}, F_{2}, G_{1}, G_{2} \in C\left[R_{+} \times R^{n} \times R^{n}, R^{n}\right]$, $F_{1}(t, 0,0)=F_{2}(t, 0,0)=G_{1}(t, 0,0)=G_{2}(t, 0,0) \equiv 0$ with $R_{+}=[0, \infty), r>0$ is a given fixed number, $t_{0} \in R_{+}$, and $\phi_{1}, \phi_{2} \in C\left([-r, 0], R^{n}\right) ; R^{n}$ denote the $n$-dimensional Euclidean space with any convenient norm $\|\cdot\|$.

We denote by $x\left(t ; t_{0}, \phi_{1}\right), y\left(t ; t_{0}, \phi_{2}\right)$ the solutions of systems (3) satisfying the initial conditions $x\left(t+t_{0}\right)=\phi_{1}(t)$, $y\left(t+t_{0}\right)=\phi_{2}(t)$. Assume that solutions $x\left(t ; t_{0}, \phi_{1}\right), y\left(t ; t_{0}, \phi_{2}\right)$ are defined on $\left[t_{0}-r, \infty\right)$ for any initial functions $\phi_{1}, \phi_{2} \in$ $C\left([-r, 0], R^{n}\right)$.

In further investigations, we need the following comparison scalar ordinary differential equations:

$$
\begin{aligned}
& u^{\prime}=f(t, u), \\
& u^{\prime}=g(t, u),
\end{aligned}
$$

and its perturbed scalar ordinary differential equation

$$
\omega^{\prime}=g(t, \omega)+q(t),
$$

where $u, \omega \in R, f, g \in R_{+} \times R \rightarrow R$, and $q \in R_{+} \rightarrow R$.

The following definitions will be needed in the sequel.

Definition 1. Letting $h_{0}, h \in \Gamma$, then

(i) $h_{0}$ is finer than $h$ if there exits a $\delta>0$ and a function $a \in \mathscr{C} \mathscr{K}$ such that

$$
h_{0}(t, x)<\delta \quad \text { implies } h(t, x) \leq a\left(t, h_{0}(t, x)\right) \text {; }
$$

(ii) $h_{0}$ is uniformly finer than $h$ if there exists a $\delta>0$ and a function $a \in \mathscr{K}$ such that

$$
h_{0}(t, x)<\delta \quad \text { implies } h(t, x) \leq a\left(h_{0}(t, x)\right) \text {. }
$$

Definition 2. The function $V(t, x, y)$ belongs to class $V_{0}$, if $V(t, x, y) \in C\left[\Omega \times R^{n} \times R^{n}, R_{+}\right], \Omega \subset R_{+}$, and $V(t, x, y)$ is Lipschitz with respect to $x$ and $y$.

Letting $V(t, x, y) \in V_{0}$, we define a derivative of the function $V(t, x, y)$ along the trajectory of systems (3) as follows:

$$
\begin{aligned}
D_{(3)} V\left(t, \phi_{1}(t), \phi_{2}(t)\right) & \\
=\limsup _{\epsilon \rightarrow 0} \frac{1}{\epsilon}\left\{V \left(t+\epsilon, \phi_{1}(t)\right.\right. & \\
& +\epsilon F_{1}\left(t, \phi_{1}(t), \max _{s \in[-r, 0]} \phi_{1}(t+s)\right), \\
& \left.\phi_{2}(t)+\epsilon F_{2}\left(t, \phi_{2}(t), \max _{s \in[-r, 0]} \phi_{2}(t+s)\right)\right) \\
& \left.-V\left(t, \phi_{1}(t), \phi_{2}(t)\right)\right\}
\end{aligned}
$$

and a derivative of the function $V(t, x, y)$ along the trajectory of systems (4) as follows:

$$
\begin{gathered}
D_{(4)} V\left(t, \phi_{1}(t), \phi_{2}(t)\right) \\
=\limsup _{\epsilon \rightarrow 0} \frac{1}{\epsilon}\left\{V \left(t+\epsilon, \phi_{1}(t)\right.\right. \\
+\epsilon\left(F_{1}\left(t, \phi_{1}(t), \max _{s \in[-r, 0]} \phi_{1}(t+s)\right)\right. \\
\left.+G_{1}\left(t, \phi_{1}(t), \max _{s \in[t-r, t]} \phi_{1}(t+s)\right)\right), \\
\phi_{2}(t)+\epsilon\left(F_{2}\left(t, \phi_{2}(t), \max _{s \in[-r, 0]} \phi_{2}(t+s)\right)\right. \\
\left.\left.\quad+G_{2}\left(t, \phi_{2}(t), \max _{s \in[t-r, t]} \phi_{2}(t+s)\right)\right)\right) \\
\left.-V\left(t, \phi_{1}(t), \phi_{2}(t)\right)\right\} .
\end{gathered}
$$

Definition 3. Letting $V \in V_{0}$ and $h_{0}, h \in \Gamma$, then $V$ is said to be

(i) relatively $h$-positive definite if there exists a $\gamma>0$ and a function $a \in \mathscr{K}$ such that

$h(t, x-y)<\delta \quad$ implies $a(h(t, x-y)) \leq V(t, x, y)$;

(ii) relatively $h_{0}$-decrescent if there exists a $\lambda>0$ and a function $b \in \mathscr{K}$ such that

$h_{0}(t, x-y)<\lambda \quad$ implies $V(t, x, y) \leq b\left(h_{0}(t, x-y)\right)$; 
(iii) weak-relatively $h_{0}$-decrescent if there exists a $\lambda>0$ and a function $b \in \mathscr{C} \mathscr{K}$ such that

$h_{0}(t, x-y)<\lambda \quad$ implies $V(t, x, y) \leq b\left(t, h_{0}(t, x-y)\right)$.

One will introduce relatively integral stability in terms of two measures for differential systems (3).

Definition 4. Letting $h_{0}, h \in \Gamma$, differential systems (3) are said to be uniform-relatively integrally stable in terms of measures $\left(h_{0}, h\right)$, if for $\alpha>0$ and any $t_{0} \geq 0$, there exists $\beta=\beta(\alpha) \in \mathscr{K}$ such that, for any initial functions $\phi_{1}, \phi_{2} \in C\left([-r, 0], R^{n}\right)$ and any perturbations $G_{1}, G_{2} \in C\left(R_{+} \times R^{n} \times R^{n}, R^{n}\right)$, the inequality

$$
h\left(t, x_{1}-y_{2}\right)<\beta, \quad t \geq t_{0}
$$

holds, provided that

$$
\begin{gathered}
\max _{s \in[-r, 0]} h_{0}\left(t_{0}+s, \phi_{1}-\phi_{2}\right)<\alpha, \\
\int_{t_{0}}^{t_{0}+T} \sup _{\left(x-y, x_{1}-y_{2}\right) \in \Omega(s, h, \beta)}\left\|G_{1}\left(s, x, x_{1}\right)-G_{2}\left(s, y, y_{2}\right)\right\| d s \leq \alpha,
\end{gathered}
$$

where $x_{1}\left(t ; t_{0}, \phi_{1}\right), y_{2}\left(t ; t_{0}, \phi_{2}\right)$ are the solutions of the initial value problem for perturbed differential systems with "maxima" (4).

\section{Main Results}

In further investigations, we need the following comparison result.

Lemma 5. Let the following conditions hold:

$\left(\mathrm{H}_{1}\right) F_{1}, F_{2} \in C\left(\left[t_{0}, T\right] \times R^{n} \times R^{n}, R^{n}\right)$, where $t_{0}, T \in R_{+}$, $t_{0}<T$;

$\left(\mathrm{H}_{2}\right) V:\left[t_{0}, T\right] \times R^{n} \times R^{n} \rightarrow R_{+}, V \in V_{0}$ and

(i) for any number $t \in\left[t_{0}, T\right]$ and any function $\psi_{1}, \psi_{2} \in C\left([t-r, t], R^{n}\right)$ such that $V\left(t, \psi_{1}(t)\right.$, $\left.\psi_{2}(t)\right)>V\left(t+s, \psi_{1}(t+s), \psi_{2}(t+s)\right)$ for $s \in[-r, 0)$ the inequality

$$
D_{(3)} V\left(t, \psi_{1}(t), \psi_{2}(t)\right) \leq g\left(t, V\left(t, \psi_{1}(t), \psi_{2}(t)\right)\right)
$$

holds, where $g \in C\left(\left[t_{0}, T\right] \times R_{+}, R_{+}\right), g(t, 0) \equiv 0$;

$\left(\mathrm{H}_{3}\right) x\left(t ; t_{0}, \phi_{1}\right), y\left(t ; t_{0}, \phi_{2}\right)$ are the solutions of the initial value problem for differential systems with "maxima" (3);

$\left(\mathrm{H}_{4}\right) u^{*}(t)=u^{*}\left(t ; t_{0}, u_{0}\right)$ is the maximal solution of (6) with initial condition $u^{*}\left(t_{0}\right)=u_{0}$, which is defined for $t \in$ $\left[t_{0}, T\right]$.

Then the inequality $\max _{s \in[-r, 0]} V\left(t_{0}+s, \phi_{1}(s), \phi_{2}(s)\right) \leq$ $u_{0}$ implies the validity of the inequality $V\left(t, x\left(t ; t_{0}, \phi_{1}\right)\right.$, $\left.y\left(t ; t_{0}, \phi_{2}\right)\right) \leq u^{*}(t), t \in\left[t_{0}, T\right]$.
Proof. Let $u_{0} \in R_{+}$and $\phi_{1}, \phi_{2} \in C\left([-r, 0], R^{n}\right)$ be such that

$$
\max _{s \in[-r, 0]} V\left(t_{0}+s, \phi_{1}(s), \phi_{2}(s)\right) \leq u_{0}
$$

Let $v_{n}(t)$ be the maximal solution of the initial value problem

$$
u^{\prime}=g(t, u)+\frac{1}{n}, \quad u\left(t_{0}\right)=u_{0}+\frac{1}{n} .
$$

Let $m(t) \in C\left(\left[t_{0}-r, T\right], R_{+}\right): m(t)=V\left(t, x\left(t ; t_{0}, \phi_{1}\right)\right.$, $\left.y\left(t ; t_{0}, \phi_{2}\right)\right)$.

Because of the fact that $u^{*}\left(t ; t_{0}, u_{0}\right)=\lim _{n \rightarrow \infty} v_{n}(t)$, it is enough to prove that for any $n$ the inequality

$$
m(t) \leq v_{n}(t), \quad t \in\left[t_{0}, T\right]
$$

holds. Then the inequality $m\left(t_{0}\right)<v_{n}\left(t_{0}\right)$ holds.

Assume that inequality (21) is not true; then there exists a point $\eta \in\left(t_{0}, T\right]: m(\eta)>v_{n}(\eta)$. Let $t^{*}=\sup \left\{t \in\left[t_{0}, T\right]\right.$ : $\left.m(s)<v_{n}(s), s \in\left[t_{0}, t\right)\right\}$. According to the assumption $t^{*}<T$, we have

$$
\begin{gathered}
m\left(t^{*}\right)=v_{n}\left(t^{*}\right), \\
m(t)<v_{n}(t), \quad t \in\left[t_{0}, t^{*}\right), \\
m(t) \geq v_{n}(t), \quad t \in\left[t^{*}, t^{*}+\delta\right),
\end{gathered}
$$

where $\delta>0$ is a small enough number. From inequality (22) it follows that

$$
m^{\prime}(t) \geq v_{n}^{\prime}(t)=g\left(t^{*}, v_{n}\left(t^{*}\right)\right)+\frac{1}{n}=g\left(t^{*}, m\left(t^{*}\right)\right)+\frac{1}{n} .
$$

From $g(t, u)+1 / n>0$ on $\left[t^{*}-r, t^{*}\right] \cap\left[t_{0}, T\right]$, it follows that the function $v_{n}(t)$ is nondecreasing on $\left[t^{*}-r, t^{*}\right] \cap\left[t_{0}, T\right]$.

Therefore $m\left(t^{*}\right)>m(s)$ for $s \in\left[t^{*}-r, t^{*}\right)$.

According to condition (i) of Lemma 5 and definition of function $m(t)$, we get $m^{\prime}\left(t^{*}\right) \leq g\left(t^{*}, m\left(t^{*}\right)\right)<g\left(t^{*}, m\left(t^{*}\right)\right)+$ $1 / n$ that contradicts (23).

Therefore inequality (21) holds and the conclusion of Lemma 5 follows.

In the following results, we will obtain sufficient conditions for uniform-relatively integral stability in terms of two measures.

\section{Theorem 6. Let the following conditions hold:}

$\left(\mathrm{A}_{1}\right) h_{0}, h \in \Gamma, h_{0}$ is uniformly finer than $h$;

$\left(\mathrm{A}_{2}\right)$ there exists $V_{1} \in V_{0}$, it is relatively $h_{0}$-decrescent and

(i) for any number $t \geq 0$ and functions $\psi_{1}, \psi_{2} \in$ $C\left([t-r, t], R^{n}\right)$ such that $V_{1}\left(t, \psi_{1}(t), \psi_{2}(t)\right)>$ $V_{1}\left(t+s, \psi_{1}(t+s), \psi_{2}(t+s)\right)$ for $s \in[-r, 0)$ and $\left(t, \psi_{1}(t), \psi_{2}(t)\right) \in S(h, \rho)$, the inequality

$$
D_{(4)} V_{1}\left(t, \psi_{1}(t), \psi_{2}(t)\right) \leq f\left(t, V_{1}\left(t, \psi_{1}(t), \psi_{2}(t)\right)\right)
$$

holds, where $f \in C\left(R_{+} \times R, R\right), \rho>0$ is a constant; 
$\left(\mathrm{A}_{3}\right)$ for any number $\mu>0$, there exists $V_{2}^{(\mu)} \in V_{0}$ such that

(ii) $b(h(t, x-y)) \leq V_{2}^{(\mu)}(t, x, y) \leq a\left(h_{0}(t, x-y)\right)$, where $a, b \in \mathscr{K}$ and $\lim _{u \rightarrow \infty} b(u)=\infty$;

(iii) for any number $t \geq 0$ and functions $\psi_{1}, \psi_{2} \in$ $C\left([-r, 0], R^{n}\right)$ such that $\left(t, \psi_{1}(t), \psi_{2}(t)\right) \in$ $S(h, \rho) \cap S^{C}\left(h_{0}, \mu\right)$ and

$$
\begin{gathered}
V_{1}\left(t, \psi_{1}(t), \psi_{2}(t)\right)+V_{2}^{(\mu)}\left(t, \psi_{1}(t), \psi_{2}(t)\right) \\
>V_{1}\left(t+s, \psi_{1}(t+s), \psi_{2}(t+s)\right) \\
\quad+V_{2}^{(\mu)}\left(t+s, \psi_{1}(t+s), \psi_{2}(t+s)\right)
\end{gathered}
$$

for $s \in[-r, 0)$, the inequality

$$
\begin{array}{r}
D_{(3)} V_{1}\left(t, \psi_{1}(t), \psi_{2}(t)\right)+D_{(3)} V_{2}^{(\mu)}\left(t, \psi_{1}(t), \psi_{2}(t)\right) \\
\leq g\left(t, V_{1}\left(t, \psi_{1}(t), \psi_{2}(t)\right)+V_{2}^{(\mu)}\left(t, \psi_{1}(t), \psi_{2}(t)\right)\right) \\
\text { holds, where } g \in C\left(R_{+} \times R, R\right), g(t, 0) \equiv 0 ;
\end{array}
$$

$\left(\mathrm{A}_{4}\right)$ the zero solution of differential equation (5) is equistable;

$\left(\mathrm{A}_{5}\right)$ the zero solution of differential equation (6) is uniformintegrally stable.

Then differential systems with "maxima" (3) are uniformrelatively integrally stable in terms of measures $\left(h_{0}, h\right)$.

Proof. Since $V_{1}(t, x, y)$ is relatively $h_{0}$-decrescent, there exist $\rho_{1} \in(0, \rho)$ and $\psi_{3} \in \mathscr{K}$ such that $h_{0}(t, x-y)<\rho_{1}$, the inequality

$$
V_{1}(t, x, y) \leq \psi_{3}\left(h_{0}(t, x-y)\right)
$$

holds.

Since $h_{0}$ is uniformly finer than $h$, there exist $\rho_{0} \in\left(0, \rho_{1}\right)$ and $\psi_{4} \in \mathscr{K}: \psi_{4}\left(\rho_{0}\right)<\rho_{1}$ such that $h_{0}(t, x-y)<\rho_{0}$ implies

$$
h(t, x-y) \leq \psi_{4}\left(h_{0}(t, x-y)\right) \text {. }
$$

Let $\alpha>0$ be a number such that $\alpha<\rho_{0}$. According to condition $\left(\mathrm{A}_{3}\right)$, there exist $V_{2}^{(\alpha)}(t, x, y)$ with Lipschitz constant $M_{2}$. Let $M_{1}$ be Lipschitz constant of the function $V_{1}(t, x, y)$.

Denote $\left(M_{1}+M_{2}\right) \alpha=\alpha_{1}$. Without loss of generality, we assume $\alpha_{1}<b(\rho)$.

From condition $\left(\mathrm{A}_{4}\right)$, it follows that there exists a $\delta_{1}=$ $\delta_{1}\left(t_{0}, \alpha_{1}\right)>0$ such that the inequality $\left|u_{0}\right|<\delta_{1}$ implies that

$$
|u(t)|<\frac{\alpha_{1}}{2}, \quad t \geq t_{0}
$$

where $u(t)$ is a solution of (5) with the initial condition $u\left(t_{0}\right)=u_{0}$.

Since $\psi_{3} \in \mathscr{K}$, there exists a $\delta_{2}=\delta_{2}\left(\delta_{1}\right)>0, \delta_{2}<\rho_{1}$ such that, for $|u|<\delta_{2}$, the inequality

$$
\psi_{3}(u)<\delta_{1}
$$

holds.
From condition $\left(\mathrm{A}_{5}\right)$, it follows that there exist $\beta_{1}=$ $\beta_{1}\left(\alpha_{1}\right) \in \mathscr{K}$ and $b(\rho)>\beta_{1} \geq \alpha_{1}$ such that, for every solution $\omega(t)$ of perturbed equation (7) with the initial condition $\omega\left(t_{0}\right)=\omega_{0}$, the inequality

$$
|\omega(t)|<\beta_{1}, \quad t \geq t_{0},
$$

holds, provided that $\left|\omega_{0}\right|<\alpha_{1}$ and for every $T>0$ : $\int_{t_{0}}^{t_{0}+T}|q(s)| d s<\alpha_{1}$.

Since $b \in \mathscr{K}, \lim _{s \rightarrow \infty} b(s)=\infty$, and $\psi_{4}(\alpha)<\psi_{4}\left(\rho_{0}\right)<$ $\rho_{1}<\rho$, we choose $\beta=\beta\left(\beta_{1}\right)>0, \rho>\beta>\alpha, \beta>\psi_{4}(\alpha)$ such that

$$
b(\beta) \geq \beta_{1} .
$$

Since $a, \psi_{4} \in \mathscr{K}$ and $\beta>\psi_{4}(\alpha)$, we can find $\delta_{3}=$ $\delta_{3}\left(\alpha_{1}, \beta\right)>0, \alpha<\delta_{3}<\min \left(\delta_{2}, \rho_{0}\right)$ such that the inequalities

$$
a\left(\delta_{3}\right)<\frac{\alpha_{1}}{2}, \quad \psi_{4}\left(\delta_{3}\right)<\beta
$$

hold.

Now let the initial function $\phi_{1}, \phi_{2} \in C\left([-r, 0], R^{n}\right)$ and perturbation $G_{1}(t, x, y), G_{2}(t, x, y)$ of the right-hand side of differential systems (4) be such that

$$
\max _{s \in[-r, 0]} h_{0}\left(t_{0}+s, \phi_{1}(s)-\phi_{2}(s)\right)<\alpha,
$$

and for every $T>0$

$$
\int_{t_{0}}^{t_{0}+T} \sup _{\left(x-y, x_{1}-y_{2}\right) \in \Omega(s, h, \beta)}\left\|G_{1}\left(s, x, x_{1}\right)-G_{2}\left(s, y, y_{2}\right)\right\| d s<\alpha .
$$

We will prove that

$$
h\left(t, x_{1}(t)-y_{2}(t)\right)<\beta, \quad t \geq t_{0} .
$$

From (28) and the choice of $\beta$, it follows that $h_{0}\left(t_{0}+\right.$ $\left.s, \phi_{1}(s)-\phi_{2}(s)\right)<\alpha<\rho_{0}$ implies that $h\left(t_{0}+s, \phi_{1}(s)-\phi_{2}(s)\right) \leq$ $\psi_{4}\left(h_{0}\left(t_{0}+s, \phi_{1}(s)-\phi_{2}(s)\right)\right)<\psi_{4}(\alpha)<\beta$; that is,

$$
h\left(t_{0}+s, \phi_{1}(s)-\phi_{2}(s)\right)<\beta, \quad s \in[-r, 0] .
$$

Suppose inequality (37) is not true. Therefore, there exists a point $t^{*}>t_{0}$ such that

$$
\begin{array}{r}
h\left(t^{*}, x_{1}\left(t^{*}\right)-y_{2}\left(t^{*}\right)\right)=\beta, \\
h\left(t, x_{1}(t)-y_{2}(t)\right)<\beta, \\
t \in\left[t_{0}-r, t^{*}\right) .
\end{array}
$$

From inequality (38) and $\beta<\rho$, it follows the validity of the inclusions

$$
\begin{gathered}
\left(t, x_{1}(t), y_{2}(t)\right) \in S(h, \rho), \\
\left(x_{1}(t)-y_{2}(t), \max _{s \in[t-r, t]}\left(x_{1}(s)-y_{2}(s)\right)\right) \in \Omega(t, h, \beta),
\end{gathered}
$$

where $t \in\left[t_{0}, t^{*}\right]$. 
Assume that $h_{0}\left(t^{*}, x_{1}\left(t^{*}\right)-y_{2}\left(t^{*}\right)\right)<\delta_{3}$; then from the choice of $\delta_{3}$ and inequality (28) it follows $h\left(t^{*}, x_{1}\left(t^{*}\right)-\right.$ $\left.y_{2}\left(t^{*}\right)\right) \leq \psi_{4}\left(h_{0}\left(t^{*}, x_{1}\left(t^{*}\right)-y_{2}\left(t^{*}\right)\right)\right) \leq \psi_{4}\left(\delta_{3}\right)<\beta$, which contradicts (38). Therefore

$$
\begin{gathered}
h_{0}\left(t^{*}, x_{1}\left(t^{*}\right)-y_{2}\left(t^{*}\right)\right)>\delta_{3}, \\
h_{0}\left(t_{0}+s, \phi_{1}(s)-\phi_{2}(s)\right)<\alpha<\delta_{3}, \quad s \in[-r, 0],
\end{gathered}
$$

and there exists a point $t_{0}^{*} \in\left(t_{0}, t^{*}\right)$ such that $\delta_{3}=$ $h_{0}\left(t_{0}^{*}, x_{1}\left(t_{0}^{*}\right)-y_{2}\left(t_{0}^{*}\right)\right)$ and $\left(t, x_{1}(t), y_{2}(t)\right) \in S(h, \beta) \cap S^{c}\left(h_{0}, \delta_{3}\right)$ for $t \in\left[t_{0}^{*}, t^{*}\right)$. Since $\beta<\rho$ and $\delta_{3}>\alpha$, it follows that

$$
\left(t, x_{1}(t), y_{2}(t)\right) \in S(h, \beta) \cap S^{c}\left(h_{0}, \alpha\right), \quad t \in\left[t_{0}^{*}, t^{*}\right] .
$$

Let $r_{1}(t)$ be the maximal solution of differential equation (5) with the initial condition $r_{1}\left(t_{0}\right)=u_{0}$, where $u_{0}=$ $\max _{s \in[-r, 0]} V_{1}\left(t_{0}+s, \phi_{1}\left(t_{0}+s\right), \phi_{2}\left(t_{0}+s\right)\right)$. From condition (i) of Theorem 6 and according to Lemma 5, we obtain

$$
V_{1}\left(t, x_{1}(t), y_{2}(t)\right) \leq r_{1}(t), \quad t \in\left[t_{0}, t^{*}\right] .
$$

From inequality (30), we obtain

$$
\begin{aligned}
u_{0} & =V_{1}\left(\xi, \phi_{1}(\xi), \phi_{2}(\xi)\right) \\
& \leq \psi_{3}\left(h_{0}\left(\xi, \phi_{1}(\xi)-\phi_{2}(\xi)\right)\right) \\
& <\psi_{3}(\alpha)<\psi_{3}\left(\delta_{2}\right)<\delta_{1},
\end{aligned}
$$

where $\xi \in\left[t_{0}-r, t_{0}\right]$.

From inequalities (29), (42), and (43), we have

$$
V_{1}\left(t, x_{1}(t), y_{2}(t)\right) \leq r_{1}(t)<\frac{\alpha_{1}}{2}, \quad t \in\left[t_{0}, t^{*}\right]
$$

or

$$
\max _{s \in[-r, 0]} V_{1}\left(t_{0}^{*}+s, x_{1}\left(t_{0}^{*}+s\right), y_{2}\left(t_{0}^{*}+s\right)\right)<\frac{\alpha_{1}}{2} .
$$

From inequality (33) and condition (ii) of Theorem 6, it follows that

$$
\begin{aligned}
& V_{2}^{(\alpha)}\left(t_{0}^{*}+s, x_{1}\left(t_{0}^{*}+s\right), y_{2}\left(t_{0}^{*}+s\right)\right) \\
& \quad \leq a\left(h_{0}\left(t_{0}^{*}+s, x_{1}\left(t_{0}^{*}+s\right)-y_{2}\left(t_{0}^{*}+s\right)\right)\right)<\frac{\alpha_{1}}{2} .
\end{aligned}
$$

Consider $V \in V_{0}$ defined by

$$
V(t, x, y)=V_{1}(t, x, y)+V_{2}^{(\alpha)}(t, x, y) .
$$

From inequalities (45) and (46) it follows that

$$
\max _{s \in[-r, 0]} V_{1}\left(t_{0}^{*}+s, x_{1}\left(t_{0}^{*}+s\right), y_{2}\left(t_{0}^{*}+s\right)\right)<\alpha_{1} .
$$

Let $t \in\left[t_{0}^{*}, t^{*}\right]$ and $\psi_{1}, \psi_{2} \in C\left([-r, 0], R^{n}\right)$ be such that

$$
\begin{gathered}
\left(t, \psi_{1}(0), \psi_{2}(0)\right) \in S(h, \beta) \cap S^{c}\left(h_{0}, \alpha\right), \\
\left(\psi_{1}(0)-\psi_{2}(0), \max _{s \in[-r, 0]}\left(\psi_{1}(s)-\psi_{2}(s)\right)\right) \in \Omega(t, h, \beta),
\end{gathered}
$$

and $V\left(t, \psi_{1}(0), \psi_{2}(0)\right)>V\left(t+s, \psi_{1}(s), \psi_{2}(s)\right), s \in[-r, 0)$.
Using Lipschitz conditions for $V_{1}, V_{2}^{(\alpha)}$ and condition (iii) of Theorem 6, we obtain

$$
\begin{aligned}
D_{(4)} V & \left(t, \psi_{1}(t), \psi_{2}(t)\right) \\
= & D_{(4)} V_{1}\left(t, \psi_{1}(t), \psi_{2}(t)\right) \\
& +D_{(4)} V_{2}^{(\alpha)}\left(t, \psi_{1}(t), \psi_{2}(t)\right) \\
\leq & g\left(t, V\left(t, \psi_{1}(t), \psi_{2}(t)\right)\right)+\left(M_{1}+M_{2}\right) \\
& \times \sup _{\left(x-y, x_{1}-y_{2}\right) \in \Omega(t, h, \beta)}\left\|G_{1}\left(t, x, x_{1}\right)-G_{2}\left(t, y, y_{2}\right)\right\| \\
= & g\left(t, V\left(t, \psi_{1}(t), \psi_{2}(t)\right)\right)+q(t) .
\end{aligned}
$$

Consider differential equation (7) where the perturbation on the right-hand side is given by

$$
\begin{array}{r}
q(t)=\left(M_{1}+M_{2}\right) \\
\quad \times \sup _{\left(x-y, x_{1}-y_{2}\right) \in \Omega(t, h, \beta)}\left\|G_{1}\left(t, x, x_{1}\right)-G_{2}\left(t, y, y_{2}\right)\right\|, \\
t \in\left[t_{0}^{*}, t^{*}\right] .
\end{array}
$$

Let $r^{*}(t)$ be the maximal solution of (7) with the initial condition $r^{*}\left(t_{0}^{*}\right)=\omega_{0}^{*}$, where $\omega_{0}^{*}=\max _{s \in[-r, 0]} V\left(t_{0}^{*}+s, x_{1}\left(t_{0}^{*}+\right.\right.$ $\left.s), y_{2}\left(t_{0}^{*}+s\right)\right)$. According to Lemma 5 , the inequality

$$
V\left(t, x_{1}(t), y_{2}(t)\right) \leq r^{*}(t), \quad t \in \Xi \cap\left[t_{0}^{*}, t^{*}\right]
$$

holds, where $\Xi \subseteq\left[t_{0}^{*}, \infty\right)$ is the interval of existence of $r^{*}(t)$.

Choose a point $T^{*}>t^{*}$ such that

$$
\int_{t_{0}^{*}}^{t^{*}} q(s) d s+\frac{1}{2}\left(T^{*}-t^{*}\right) q\left(t^{*}\right)<\alpha_{1} .
$$
by

Now define the continuous function $q^{*}(t):\left[t_{0}^{*}, \infty\right) \rightarrow R$

$$
q^{*}(t)= \begin{cases}q(t), & t \in\left[t_{0}^{*}, t^{*}\right], \\ \frac{q\left(t^{*}\right)}{t^{*}-T^{*}}, & t \in\left[t^{*}, T^{*}\right], \\ 0, & t \geq T^{*} .\end{cases}
$$

From the choice of the perturbation $G_{1}(t, x, y), G_{2}(t, x, y)$ it follows that for every $T>0$ the inequality

$$
\begin{gathered}
\int_{t_{0}^{*}}^{t_{0}^{*}+T} q^{*}(s) d s \leq\left(M_{1}+M_{2}\right), \\
\int_{t_{0}}^{t_{0}+T} \sup _{\left(x-y, x_{1}-y_{2}\right) \in \Omega(s, h, \beta)}\left\|G_{1}\left(s, x, x_{1}\right)-G_{2}\left(s, y, y_{2}\right)\right\| d s<\alpha_{1}
\end{gathered}
$$

holds.

Let $r_{1}(t)$ be the maximal solution of (7) with the initial condition $r_{1}\left(t_{0}^{*}\right)=\omega_{0}^{*}$, where the perturbation of the righthand side is defined above function $q^{*}(t)$. Note that $r_{1}(t)=$ $r^{*}\left(t, t_{0}^{*}, \omega_{0}^{*}\right), t \in\left[t_{0}^{*}, t^{*}\right)$. 
From inequality (48) it follows that $\left|\omega_{0}^{*}\right|<\alpha_{1}$ and therefore inequality (31) holds; that is,

$$
r_{1}(t)<\beta_{1}, \quad t \geq t_{0}^{*} .
$$

From inequalities (52) and (56), the choice of the point $t^{*}$, and condition (iii), we obtain

$$
\begin{aligned}
b(\beta) & \geq \beta_{1}>r_{1}\left(t^{*}\right)=r^{*}\left(t^{*}\right) \\
& \geq V\left(t^{*}, x_{1}\left(t^{*}\right), y_{2}\left(t^{*}\right)\right) \\
& \geq V_{2}^{(\alpha)}\left(t^{*}, x_{1}\left(t^{*}\right), y_{2}\left(t^{*}\right)\right) \\
& \geq b\left(h\left(t^{*}, x_{1}\left(t^{*}\right)-y_{2}\left(t^{*}\right)\right)\right) \\
& =b(\beta) .
\end{aligned}
$$

The obtained contradiction proves the validity of inequality (37) for $t \geq t_{0}$.

Inequality (37) proves uniform-relatively integral stability in terms of measures $\left(h_{0}, h\right)$ of the considered differential systems with "maxima."

The following example is an application of Theorem 6.

Example 1. Consider the two differential systems with "maxima"

$$
\begin{gathered}
x^{\prime}=-x-x e^{t-t_{0}}+\frac{1}{2} \max _{s \in[t-r, t]} x(s), \quad t \geq t_{0}, \\
x\left(t+t_{0}\right)=\phi_{1}(t), \quad t \in[-r, 0], \\
y^{\prime}=-y e^{t-t_{0}}+\frac{1}{2} \max _{s \in[t-r, t]} y(s), \quad t \geq t_{0} \\
y\left(t+t_{0}\right)=\phi_{2}(t), \quad t \in[-r, 0]
\end{gathered}
$$

and the perturbed systems

$$
\begin{aligned}
x^{\prime}= & -x-x e^{t-t_{0}}+\frac{1}{2} \max _{s \in[t-r, t]} x(s) \\
& +\frac{1}{2} \max _{s \in[t-r, t]} x(s) e^{-t}, \quad t \geq t_{0}, \\
x(t+ & \left.t_{0}\right)=\phi_{1}(t), \quad t \in[-r, 0], \\
y^{\prime}= & -y e^{t-t_{0}}+\frac{1}{2} \max _{s \in[t-r, t]} y(s) \\
& +\frac{1}{2} \max _{s \in[t-r, t]} y(s) e^{-t}, \quad t \geq t_{0}, \\
y(t+ & \left.t_{0}\right)=\phi_{2}(t), \quad t \in[-r, 0] .
\end{aligned}
$$

Let $V_{1}(t, x, y)=x^{2}+y^{2}, V_{2}^{(\mu)}(t, x, y)=x^{2}+2 y^{2}$, and $h_{0}(t, x-y)=\sqrt{2}(\|x\|+\|y\|), h(t, x-y)=\sqrt{2\left(x^{2}+y^{2}\right)}$.

Using the inequality $\sqrt{x^{2}+y^{2}} \leq\|x\|+\|y\|$, it is easy to check the validity of the conditions $\left(\mathrm{A}_{1}\right)$ and (ii) of Theorem 6 for $a(u)=u^{2}, b(u)=(1 / 2) u^{2}$.
Letting $\psi_{1}, \psi_{2} \in C\left([t-r, t], R^{n}\right)$ be such that $\psi_{1}^{2}(t)>$ $\psi_{1}^{2}(t+s), \psi_{2}^{2}(t)>\psi_{2}^{2}(t+s), s \in[-r, 0), t \geq t_{0}$, then $V_{1}\left(t, \psi_{1}(t), \psi_{2}(t)\right)>V_{1}\left(t+s, \psi_{1}(t+s), \psi_{2}(t+s)\right)$ and

$$
\begin{aligned}
D_{(59)} V_{1}\left(t, \psi_{1}(t), \psi_{2}(t)\right) & \\
= & 2 \psi_{1}(t) \psi_{1}(t)^{\prime}+2 \psi_{2}(t) \psi_{2}(t)^{\prime} \\
= & 2 \psi_{1}(t)\left\{-\psi_{1}(t)-\psi_{1}(t) e^{t-t_{0}}+\frac{1}{2} \max _{s \in[t-r, t]} \psi_{1}(s)\right. \\
& \left.+\frac{1}{2} \max _{s \in[t-r, t]} \psi_{1}(s) e^{-t}\right\} \\
& +2 \psi_{2}(t)\left\{-\psi_{2}(t) e^{t-t_{0}}+\frac{1}{2} \max _{s \in[t-r, t]} \psi_{2}(s)\right. \\
& \left.+\frac{1}{2} \max _{s \in[t-r, t]} \psi_{2}(s) e^{-t}\right\} \\
\leq & \left(-2 e^{t-t_{0}}+1+e^{-t}\right)\left(\psi_{1}^{2}(t)+\psi_{2}^{2}(t)\right) \\
\leq & e^{-t}\left(\psi_{1}^{2}(t)+\psi_{2}^{2}(t)\right) .
\end{aligned}
$$

Letting $f(t, u)=e^{-t} u$, then $D_{(57)} V_{1}\left(t, \psi_{1}(t), \psi_{2}(t)\right) \leq$ $f\left(t, V_{1}\left(t, \psi_{1}(t), \psi_{2}(t)\right)\right)$.

Considering the comparison scalar differential system

$$
u^{\prime}=e^{-t} u, \quad u_{0}=u\left(t_{0}\right),
$$

the solution is $u=u_{0} e^{e^{-t_{0}}} e^{-e^{-t}}, t \geq t_{0}$, and we can prove that the solution is equistable; that is, the conditions $\left(\mathrm{A}_{2}\right)$ and $\left(\mathrm{A}_{4}\right)$ of Theorem 6 hold.

For $s \in[-r, 0), t \geq t_{0}$, the inequality

$$
\begin{aligned}
D_{(58)} & V_{1}\left(t, \psi_{1}(t), \psi_{2}(t)\right)+D_{(58)} V_{2}^{(\mu)}\left(t, \psi_{1}(t), \psi_{2}(t)\right) \\
= & 4 \psi_{1}(t) \psi_{1}(t)^{\prime}+6 \psi_{2}(t) \psi_{2}(t)^{\prime} \\
= & 4 \psi_{1}(t)\left\{-\psi_{1}(t)-\psi_{1}(t) e^{t-t_{0}}+\frac{1}{2} \max _{s \in[t-r, t]} \psi_{1}(s)\right\} \\
& +6 \psi_{2}(t)\left\{-\psi_{2}(t) e^{t-t_{0}}+\frac{1}{2} \max _{s \in[t-r, t]} \psi_{2}(s)\right\} \\
\leq & \left(-2 e^{t-t_{0}}+1\right)\left(2 \psi_{1}^{2}(t)+3 \psi_{2}^{2}(t)\right) \\
\leq & 0
\end{aligned}
$$

holds. From (62), the inequality

$$
\begin{aligned}
D_{(58)} & V_{1}\left(t, \psi_{1}(t), \psi_{2}(t)\right)+D_{(58)} V_{2}^{(\mu)}\left(t, \psi_{1}(t), \psi_{2}(t)\right) \\
& \leq g\left(t, V_{1}\left(t, \psi_{1}(t), \psi_{2}(t)\right)+V_{2}^{(\mu)}\left(t, \psi_{1}(t), \psi_{2}(t)\right)\right)
\end{aligned}
$$

holds, where $u^{\prime}=g(t, u) \equiv 0$ and its perturbed differential equation $\omega^{\prime}=0+\omega_{0} e^{-t}$. We can prove that the differential equation $g(t, u)=0$ is uniform-integrally stable. So the conditions $\left(\mathrm{A}_{3}\right)$ and $\left(\mathrm{A}_{5}\right)$ of Theorem 6 hold.

According to Theorem 6, differential systems with "maxima" (58) are uniform-relatively integrally stable in terms of two measures $\left(h_{0}, h\right)$. 


\section{Conflict of Interests}

The authors declare that they have no conflict of interests.

\section{Authors' Contribution}

All authors completed the paper together. All authors read and approved the final paper.

\section{Acknowledgments}

The authors would like to thank the reviewers for their valuable suggestions and comments. This paper is supported by the National Natural Science Foundation of China (11271106) and the Natural Science Foundation of Hebei Province of China (A2013201232).

\section{References}

[1] D. D. Bainov and S. G. Hristova, Diffierential Equations with Maxima, Taylor \& Francis, 2011.

[2] S. G. Hristova, "Razumikhin method and cone valued Lyapunov functions for impulsive differential equations with 'supremum," International Journal of Dynamical Systems and Differential Equations, vol. 2, no. 3-4, pp. 223-236, 2009.

[3] A. D. Mishkis, "On some problems of the theory of diffierential equations with deviating argument," Russian Mathematical Surveys, vol. 32, no. 2, pp. 181-210, 1977.

[4] P. González and M. Pinto, "Convergent solutions of certain nonlinear differential equations with maxima," Mathematical and Computer Modelling, vol. 45, no. 1-2, pp. 1-10, 2007.

[5] I. Vrkoc, "Integral stability," Czechoslovak Mathematical Journal, vol. 9, no. 84, pp. 71-129, 1959.

[6] V. Lakshmikantham and S. Leela, Diffierential and Integral Inequalities, vol. 1, Academic Press, New York, NY, USA, 1969.

[7] A. A. Soliman and M. H. Abdalla, "Integral stability criteria of nonlinear differential systems," Mathematical and Computer Modelling, vol. 48, no. 1-2, pp. 258-267, 2008.

[8] S. Hristova, "Integral stability in terms of two measures for impulsive differential equations with 'supremum,' Communications on Applied Nonlinear Analysis, vol. 16, no. 3, pp. 37-49, 2009.

[9] S. G. Hristova, "Integral stability in terms of two measures for impulsive functional differential equations," Mathematical and Computer Modelling, vol. 51, no. 1-2, pp. 100-108, 2010. 


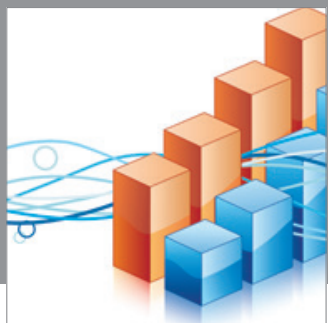

Advances in

Operations Research

mansans

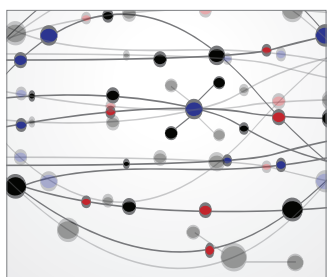

The Scientific World Journal
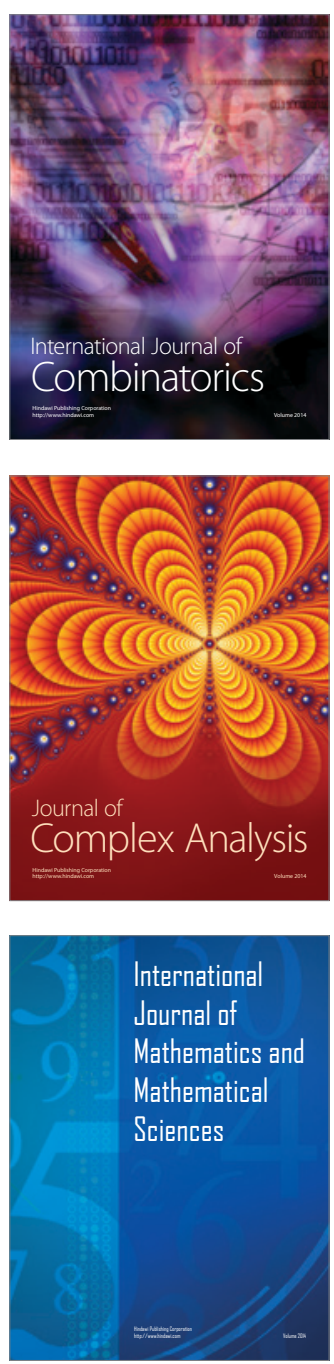
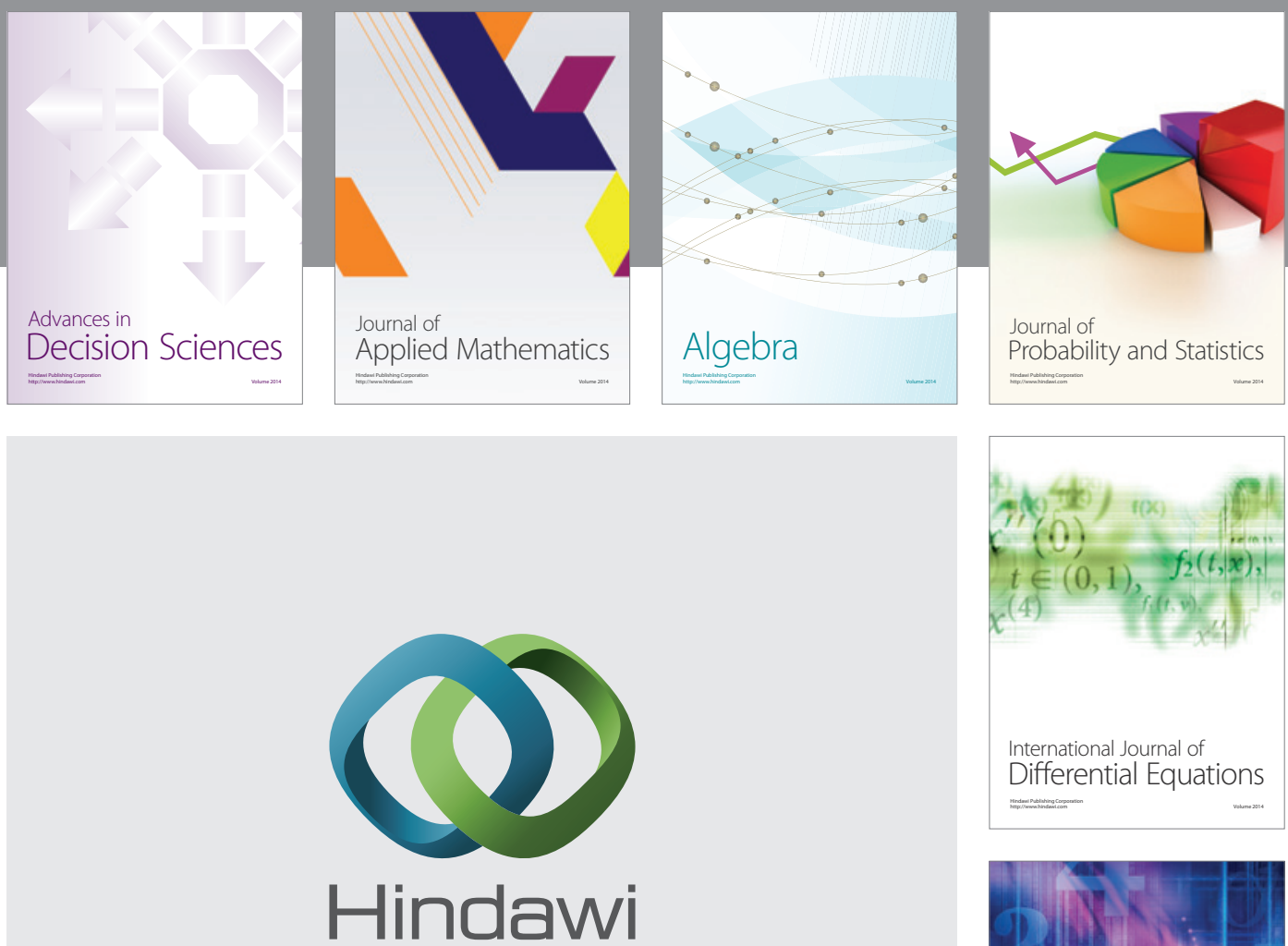

Submit your manuscripts at http://www.hindawi.com
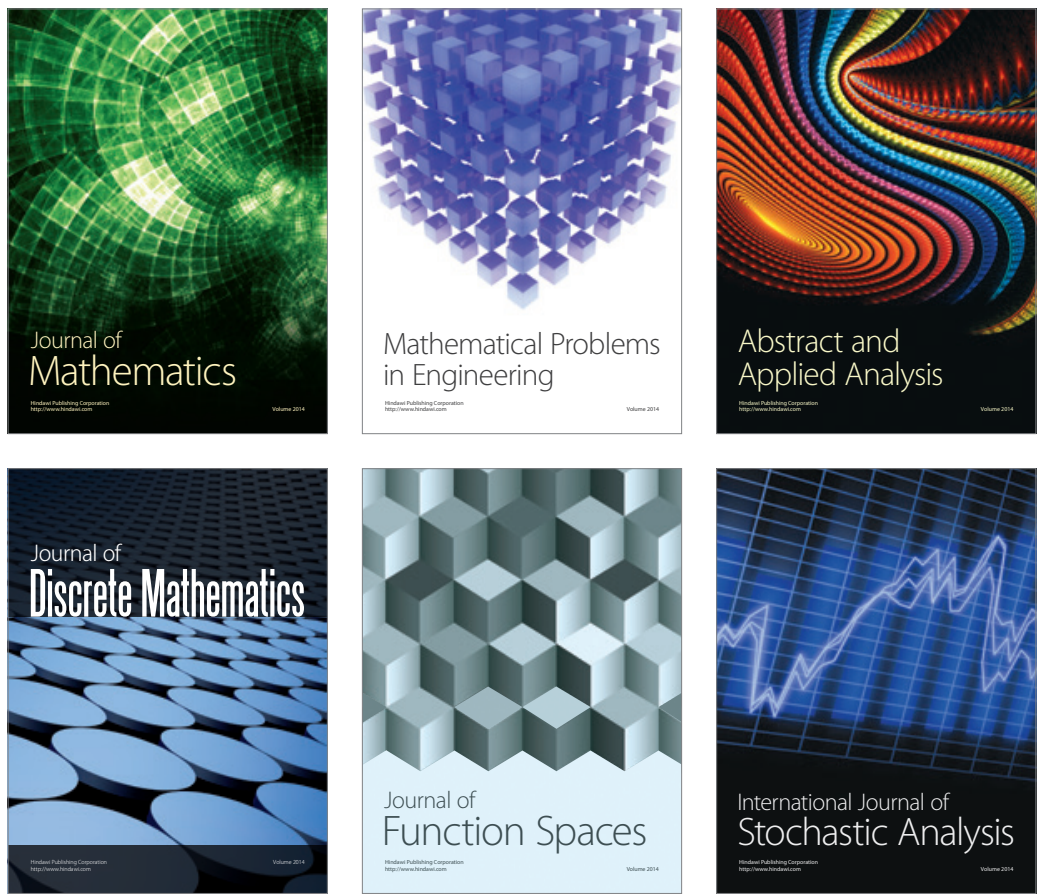

Journal of

Function Spaces

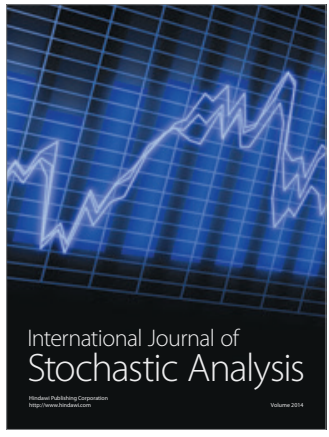

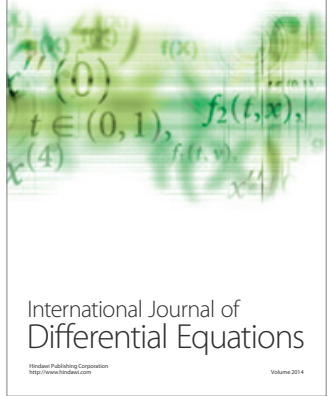
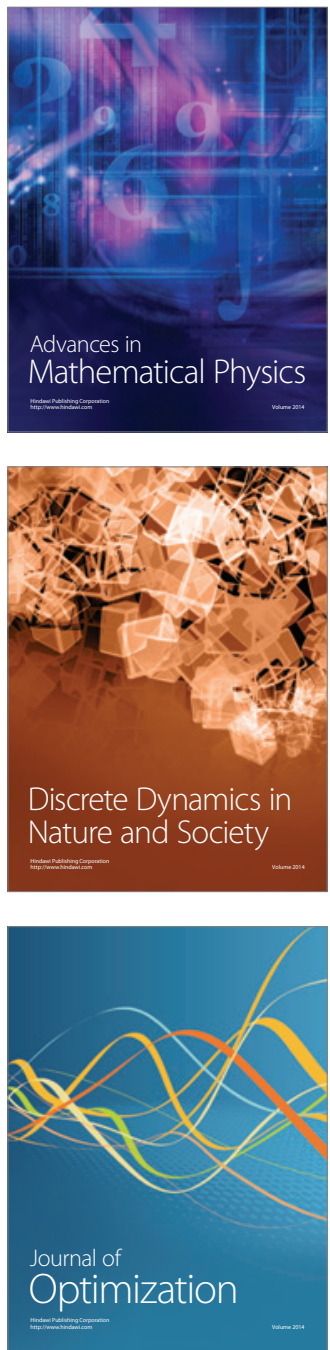